\title{
Handicap vestibulaire
}

\author{
Jean-Philippe Guyot
}

Ce numéro d'ORL Nova rapporte quelques-unes des conférences présentées lors du XXXIIIe Symposium de la Société d'Otoneurologie de Langue française, tenu à Genève en mai 1999.

La recherche fondamentale et les études cliniques améliorent constamment nos connaissances de la physiologie et de la physiopathologie vestibulaires. Le raffinement des techniques d'investigation permet une approche diagnostique toujours plus précise des vertiges et des troubles de l'équilibre. Toutefois, l'évaluation de la fonction vestibulaire, et plus globalement de l'équilibre, reste limitée. Les résultats des tests électrophysiologiques ne corroborent pas toujours l'importance des plaintes et des troubles des patients. Il est notoire que les patients avec une aréflexie vestibulaire unilatérale peuvent ne pas se plaindre alors que d'autres, avec un bilan normal, se plaignent amèrement de déséquilibre. De plus, les tests électrophysiologiques ne donnent aucune indication de la gêne ressentie par les patients souffrant d'une affection otoneurologique. Or, les troubles de l'équilibre et les épisodes de vertige entraînent des conséquences socio-professionnelles non négligeables chez la plupart des patients et peuvent représenter une expérience traumatisante, à l'origine de perturbations psychologiques qui nécessitent un diagnostic et une prise en charge adéquate. C'est pourquoi nous avons souhaité que ce symposium soit consacré à discuter des développements les plus récents dans l'évaluation des troubles de l'équilibre ainsi que, et essentiellement, des conséquences physiques et psychiques des affections vestibulaires, concept que nous proposons de désigner comme le «handicap vestibulaire».

Ainsi, la prise en charge thérapeutique des patients souffrant d'affections otoneurologiques est en train de changer. Elle n'est plus limitée à la prescription de médicaments ou à un geste chirurgical. Les otoneurologues ont bien compris la dualité physique et psychique des affec- tions qu'ils traitent. Cette dualité est abordée d'une façon originale lors de consultations conjointes médico-psychologiques (CCMP). Il ne s'agit plus d'une prise en charge multidisciplinaire, où le malade est référé par un somaticien à un psychiatre, mais d'une approche interdisciplinaire réunissant deux thérapeutes, un somaticien et un psychologue, qui reçoivent en même temps le patient pour aborder d'emblée avec lui tous les aspects de son affection, somatiques et psycho-sociaux. La CCMP génère des interactions fructueuses pour le patient et représente pour le somaticien une occasion unique de voir comment aborder les problèmes psychiques liés ou consécutifs aux affections otoneurologiques.

En raison de la complexité des affections otoneurologiques et de leurs répercussions psychiques, sociales et affectives, la mesure du handicap vestibulaire est difficile. Certes, toutes sortes de tests visant à mieux évaluer la fonction vestibulaire sont en cours de développement. Les seules mesures de la réponse du canal latéral aux stimulations caloriques et pendulaires sont bien lointaines, bien qu'encore très utiles. Aujourd'hui, il est possible d'étudier la fonction vestibulaire en réponse à des stimulations physiologiques, d'évaluer la fonction des organes otolithiques, d'enregistrer des réponses myogéniques d'origine sacculaire, d'analyser finement les réflexes vestibulo-spinaux dans toutes sortes de conditions. Toutefois, les otoneurologues ont compris que ces mesures sont encore insuffisantes et qu'il est nécessaire de développer des outils de mesure du handicap vestibulaire. C'est ainsi que divers questionnaires spécifiques ont été élaborés et sont aujourd'hui en cours d'évaluation.

Les acouphènes représentent une autre affection chronique qu'il convient de considérer dans sa dualité, physique et psychique. La relation entre les deux pôles de l'affection est toutefois différente de celle observée en cas de vertiges. L'intolérance aux acouphènes est souvent

\author{
KARGER \\ Fax + 41613061234 \\ E-Mail karger@karger.ch \\ www. karger.com \\ 구 2000 S. Karger AG, Basel \\ Accessible online at: \\ www.karger.com/journals/orn
}


l'indice d'un état dépressif latent ou non reconnu. En raison de l'absence de traitement efficace, le patient devra développer des processus d'acceptation tout à fait spécifiques. Dans cette situation, la prise en charge dans une CCMP s'avère efficace pour aider le patient à mettre en place des processus qu'il ignorait jusque-là.

Ce symposium a montré que le champ de connaissances de l'otoneurologie ne cessait de s'élargir, impliquant des domaines aussi variés que l'oto-rhino-laryngologie, la neurologie, la microchirurgie de l'oreille et de la base du crâne, des domaines techniques tels que l'électrophysiologie et l'informatique, domaines auxquels s'ajoute maintenant la capacité à entendre, à reconnaître et à prendre en charge les répercussions psychologiques et socio-professionnelles des affections. Il n'était que de voir l'enthousiasme des participants au symposium de Genève pour comprendre combien les multiples facettes de cette discipline médico-chirurgicale la rendent fascinante. Nous souhaitons transmettre notre enthousiasme aux lecteurs de ce numéro d'ORL Nova.

\section{Remerciements}

L'organisation d'un Symposium réunissant une centaine de participants représente une somme de travail non négligeable, mais qui est très largement compensée par l'occasion unique d'établir des contacts privilégiés avec les collègues et de renforcer des liens d'amitié. Je tiens à remercier l'ensemble des participants au Symposium ainsi que tous ceux qui m'ont aidé à préparer cette manifestation, en particulier le Dr Pierre Liard, co-organisateur de cette manifestation. 